\title{
Aromatic L-amino acid decarboxylase deficiency: perspectives on diagnosis and management
}

This article was published in the following Dove Press journal:

Pediatric Health, Medicine and Therapeutics

18 August 2013

Number of times this article has been viewed

\author{
Stacey KH Tay ${ }^{1,2}$ \\ Furene SJ Wang ${ }^{2}$ \\ Jeremy BY Lin² \\ 'Department of Pediatrics, Yong Loo \\ Lin School of Medicine, National \\ University of Singapore, Singapore; \\ ${ }^{2}$ Khoo Teck Puat-National University \\ Children's Medical Institute, National \\ University Hospital, Singapore
}

\begin{abstract}
Aromatic L-amino acid decarboxylase (AADC) deficiency is an autosomal recessive neurotransmitter defect that results in a combined central deficiency of monoamines, namely catecholamines and serotonin. To date, less than 100 patients have been described with AADC deficiency, most with a severe neurological phenotype. This review article illustrates the pathophysiology, diagnostic methods, and therapeutic strategies for patients with AADC deficiency. Two patients in our institution have extremely mild phenotypes and their robust response to treatment illustrates the variable severity of this condition and the need for early diagnosis of this potentially treatable disease.
\end{abstract}

Keywords: aromatic amino acid decarboxylase deficiency, AADC deficiency, dopa decarboxylase gene, $D D C$ gene, dopamine deficiency, neurotransmitter disorder

\section{Background and pathophysiology}

Deficiency of aromatic L-amino acid decarboxylase (AADC) is associated with severe developmental delay, oculogyric crises (OGCs), and autonomic dysfunction. It is an inborn error of metabolism that affects serotonin and dopamine biosynthesis. ${ }^{1,2}$

The enzyme L-amino acid decarboxylase converts L-3,4-dihydroxyphenylalanine (L-dopa) to dopamine and 5-hydroxytryptophan (5HTP) to serotonin (Figure 1A). Thus, lack of the enzyme leads to a deficiency of these neurotransmitters and to significant episodic and chronic neurological problems.

Dopamine and serotonin play key roles in the brain, and are involved in the control of locomotion, mood, and behavior. Dopamine is involved in the control of motor function, and a decrease in striatal concentrations can lead to extrapyramidal manifestations. ${ }^{3}$ Mapping studies have shown that dopamine has a much wider distribution beyond the basal ganglia, suggesting a more complex and extended role in the brain. ${ }^{4}$ Dopamine also has important roles in several physiological functions, such as control of voluntary locomotion, cognitive processes, neuroendocrine secretion (prolactin), and behaviors (such as through the reward mechanism). ${ }^{5}$

Similarly, serotonin plays a role (albeit less than dopamine) in motor control. ${ }^{6}$ Serotonergic innervation is important for sensory stimuli processing in the cerebral cortex and for the regulation of the sleep-wake cycle. It also plays a part in autonomic control of respiration, temperature, and mood. The serotonin syndrome, ${ }^{7}$ which is the result of excessive central serotonin activity that is usually due to drugs, may result in dystonia, myoclonus, and tremor, in addition to cognitive and autonomic symptoms.
Correspondence: Stacey KH Tay Department of Paediatrics, National University of Singapore,

IE Kent Ridge Road, Singapore 119228

Tel +6567724420

Fax +6567797486

Email stacey_tay@nuhs.edu.sg 
A

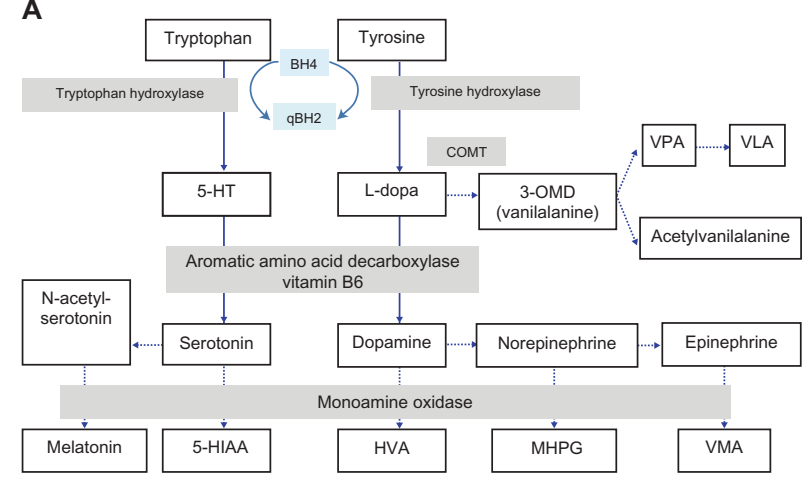

B

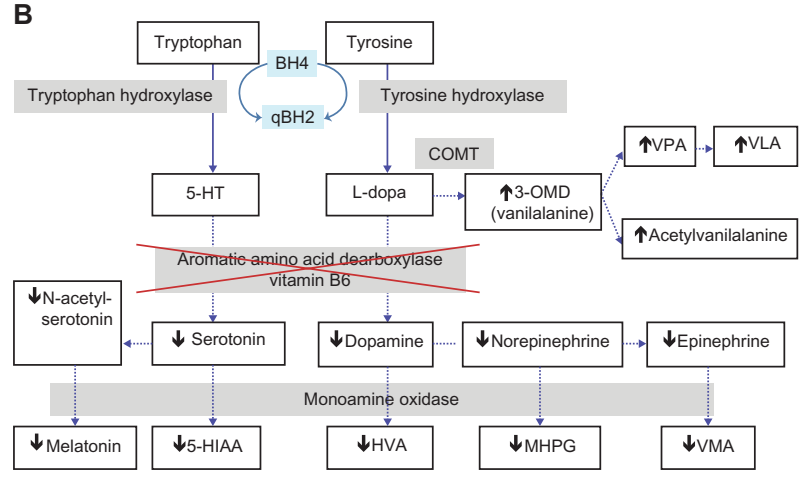

Figure I Biosynthetic pathway of dopamine, catecholamines, and melatonin. Notes: (A) Biosynthetic pathway of dopamine, catecholamines and melatonin. Enzymes and cofactors are shown in the shaded boxes. (B) Effect of AADC deficiency where 5-HIAA, HVA, and MHPG levels are significantly reduced in the CSF. Moreover, 5-HT and 3-OMD would be elevated. $\mathrm{N}$-acetyl tyrosine, VPA, and acetylvanilalanine are other metabolites that can be detected by urine organic acid analysis.

Abbreviations: $\mathrm{BH} 4$, tetrahydrobiopterin; $\mathrm{qBH} 2$, quinonoid dihydrobiopterin; COMT, catechol-O-methyltransferase; 5-HT, 5-hydroxytryptophan; L-dopa, L-3,4dihydroxyphenylalanine; 3-OMD, 3-O-methyldopa; VPA, vanilpyruvic acid; VLA, vanillactic acid; 5-HIAA, 5-hydoxyindolacetic acid; HVA, homovanillic acid; MHPG, 3-methoxy-4-hydroxyphenylglycol; VMA, vanillylmandelic acid; AADC, aromatic amino-acid decarboxylase; CSF, cerebrospinal fluid.

Decreased AADC levels would result in a block of both dopamine and serotonin synthesis (Figure 1A). Serotonin and dopamine deficiency would be responsible for severe hypotonia and the inability to program and perform voluntary motor movements. Serotonin deficiency would affect appetite, sleep, memory, learning, body temperature, mood, cardiovascular function, and endocrine functions. Behavioral disturbances, such as disinhibited behavior, have also been observed. ${ }^{8}$ Noradrenaline and adrenaline levels affect attention, mood, sleep, cognitive ability, and stress hormones. Adrenaline and noradrenaline deficiency would account for the temperature instability and excessive sweating of patients with AADC deficiency. ${ }^{8}$

Thus, AADC-deficient patients would typically present with difficulties in maintaining motor movements, dystonia, sleep, OGCs, autonomic dysfunction such as drooling, temperature dysregulation, as well as memory and learning difficulties. Since AADC deficiency is a central neurotransmitter disorder, fluctuations of symptoms may occur and occasionally be observed to be ameliorated by rest and sleep, which allows for the replenishing of the neurotransmitters.

\section{Epidemiology and prevalence}

Since AADC deficiency was first reported in 1990, fewer than 100 patients with AADC deficiency have been reported worldwide. So far, cases have presented in patients aged 4 months to 24 years old. Most of the patients presented during infancy and childhood. ${ }^{9}$ There appears to be a significant preponderance of Southern Chinese individuals with AADC deficiency (about onethird of known cases in the JAKE database, ${ }^{10}$ which is an international database of patients with AADC deficiency). This may be due to a possible founder mutation, a splicing mutation IVS6 $+4 \mathrm{~A}>\mathrm{T}$ present in Chinese patients from Taiwan, Singapore, and Malaysia (likely migrants from Southern China).

\section{Clinical phenotype}

AADC deficiency was first described by Hyland and Clayton ${ }^{11}$ in 1990 in a pair of male monozygotic twins who presented with extreme hypotonia and OGCs. Central and peripheral concentrations of biogenic amines and their metabolites were reduced significantly. Specifically, cerebrospinal fluid (CSF) concentrations of homovanillic acid (HVA) and 5-hydroxyindoleacetic acid (5HIAA) were decreased, as well as whole-blood serotonin and plasma catecholamines. Pterin and phenylalanine metabolism was normal. A liver biopsy showed absent activity of AADC.

Most patients with AADC deficiency present in infancy or early childhood with severe symptoms of hypotonia, OGCs, and marked developmental delay. ${ }^{12}$ The two main characteristics at presentation are hypotonia and OGCs. Movement disorders such as dystonia, chorea, and hypokinesia were also common. While hypotonia may not be a very specific feature, this particular combination with movement disorders, OGCs, and an episodic fluctuation of symptoms would be a strong indicator of a central neurotransmitter disorder such as AADC deficiency (Figure 2). ${ }^{13}$ Other manifestations included ptosis, poor visual fixation, sleeping difficulties, autonomic dysfunction, temperature instability, irritability, and nasal congestion.

Autonomic dysfunction in AADC deficiency is characterized by a significant impairment of sympathetic regulation of heart rate and blood pressure. Autonomic testing has been performed for two previously diagnosed cases. ${ }^{11}$ 


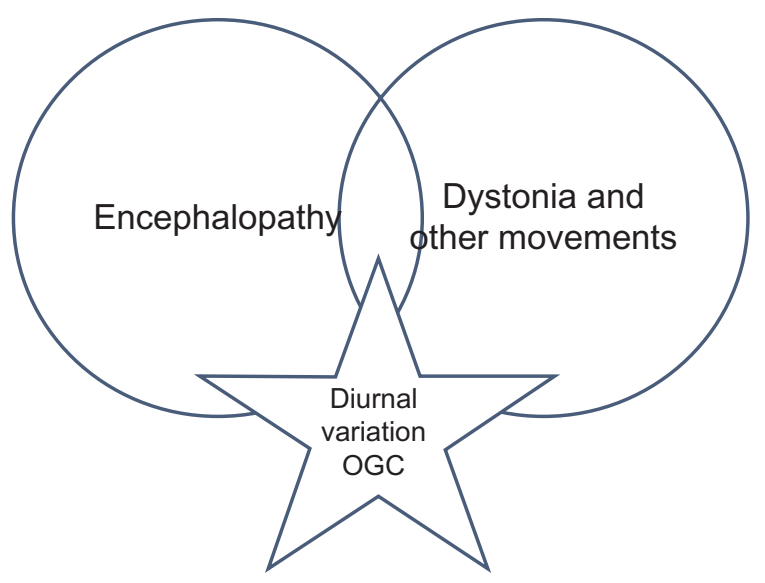

Figure 2 Diagram showing the overlap of typical symptoms of AADC, with encephalopathic state, dystonia, and other movement disorders, as well as the distinguishing features of fluctuating symptoms during the day, possible diurnal variation, and presence of OGC

Abbreviation: OGC, oculogyric crises.

Electrocardiogram, instantaneous lung volume, and blood pressure monitoring were studied. The recordings of average heart rate, arterial pressure, and respiratory response were obtained during infusions of isoproterenol, nitroprusside, esmolol, phenylephrine, and after atropine administration. Severe impairment of sympathetic blood pressure modulation was found in both patients.

Other investigations such as magnetic resonance imaging of the brain usually do not reveal any specific abnormalities; however, up to one-quarter of patients may have cerebral atrophy, degenerative white matter changes, abnormal myelination, and thinning of the corpus callosum. ${ }^{9,14}$ Seizures are rare and there is no particular distinctive electroencephalography pattern associated with AADC deficiency.

\section{Differential diagnoses}

The combination of pyramidal, extrapyramidal, and autonomic symptoms may suggest differentials such as an encephalopathy due to a diffuse process, including infective or metabolic causes. Hypotonia, ptosis, and decreased motor movements may raise the suspicion of a neuromuscular disorder such as congenital myasthenia, or metabolic disorders like mitochondrial encephalopathies. Prolonged OGCs and dystonia may raise the suspicion of seizures. Early evolving (hypotonic phase) or dyskinetic cerebral palsy, other earlyonset disorders of dopamine metabolism, and hyperekplexia (excessive startle response) are other possible differential diagnoses. ${ }^{13}$

Besides the primary monoamine neurotransmitter defects, including AADC deficiency, there are also secondary causes of monoamine neurotransmitter defects. These occur in various other neurological conditions such as mitochondrial diseases, epileptic encephalopathies, pontocerebellar hypoplasia, perinatal asphyxia, opsoclonus-myoclonus, leukodystrophies, and Rett syndrome.

It is imperative to differentiate between the various primary neurotransmitter defects, since treatment is different in each of them. Defects in aromatic amino acid metabolism lead to the deficiencies of dopamine and serotonin in the central nervous system. The plasma, urine, or CSF levels of the metabolites (phenylalanine, tetrahydrobiopterin $\left[\mathrm{BH}_{4}\right]$, 7,8-dihydrobiopterin, neopterin, sepiapterin, primapterin, HVA, 5HIAA, 3-O-methyldopa [3-OMD]) enable the clinching of the diagnosis.

Inherited abnormalities of vitamin B6 metabolism can mimic AADC deficiency biochemically. The clinical features are dissimilar, as affected children present with severe anticonvulsant-resistant neonatal epileptic encephalopathy, often with prenatal onset of intractable seizures. Autosomal recessive pyridoxamine $5^{\prime}$-phosphate oxidase deficiency, for example, leads to a decrease in the synthesis of pyridoxal phosphate from pyridoxine and pyridoxamine, which results in a secondary impairment of dopamine and serotonin production.

The clinical features of AADC deficiency are also reminiscent of features of children with $\mathrm{BH}_{4}$ deficiency states such as 6-pyruvovyl-tetrahydropterin synthase deficiency, dihydropteridine reductase deficiency, as well as the autosomal recessive form of guanosine triphosphate cyclohydrolase deficiency. These are pediatric neurotransmitter disorders that may also present with an encephalopathic state, movement disorders such as dystonia, as well as chorea and tremor, epilepsy, and developmental delay.

\section{Biochemical analysis}

AADC is a single enzyme that decarboxylates both L-dopa and 5HTP. Thus, the enzyme defect is expected to impair the synthesis of both dopamine and serotonin. CSF would show low levels of 5-HIAA, HVA, and 3-methoxy-4hydroxyphenylglycol, and increased levels of L-dopa, 3-OMD, and 5HTP if there were a block at the level of AADC (Figure 1B). Pterin levels would be normal.

The initial steps in the synthesis of both dopamine and serotonin involve the hydroxylation of tyrosine to form L-dopa, which is catalyzed by tyrosine hydroxylase, and the hydroxylation of tryptophan to 5HTP in a reaction catalyzed by tryptophan hydrolase. Both hydroxylation reactions require $\mathrm{BH}_{4}$ as a cofactor. Following that, the next step in the pathway is the decarboxylation of L-dopa and 5-HTP by a single 
vitamin B6-requiring enzyme, AADC, to form dopamine and serotonin, respectively. Pyridoxine (vitamin B6) is a critical cofactor in AADC activity. Pyridoxal phosphate is the active metabolite of pyridoxine.

The major diagnostic metabolite is 3-OMD, the methylated product of L-dopa; 3-OMD is very stable and thus has a long half-life in plasma and CSF. It is important to compare the 3-OMD concentrations with age-related reference values because the 3-OMD concentrations drop rapidly during the first 6 months after birth.

CSF may not always be available for testing, but there are other measurable metabolites in plasma or urine. Specifically, 3-OMD, 5HTP, and L-dopa can be detected by their natural fluorescence. These compounds can be easily measured in plasma and urine. The major metabolite of 3-OMD is vanillactic acid (VLA). Analysis of urine organic acids can reveal an increased concentration of VLA in AADC deficiency. ${ }^{15}$ Major obstacles, however, to using VLA as a screening test include the fact that some AADC deficient patients do not have high urinary VLA (only mildly elevated), ${ }^{9}$ and furthermore, many major laboratories do not include VLA in their routine organic acid analysis. ${ }^{1}$

The paradoxical hyperdopaminuria in AADC deficiency was an observation that was a puzzle until recently.,16 Wassenberg et $\mathrm{al}^{17}$ noted that the hyperdopaminuria may be due to increased AADC activity in the proximal tubular epithelial cells compared to other tissues such as the brain. As plasma L-dopa levels are relatively high in AADC-deficient patients, urine dopamine levels tend to be elevated as the renal AADC pathway is the only metabolic pathway for forming renal dopamine. Because of this, urine neurotransmitter profiles should be evaluated with caution and the CSF neurotransmitter profile remains central to the diagnosis of patients with AADC deficiency.

Although an elevation of VLA has been described as the only abnormality detected in the organic acid analysis of urine, increased levels of vanilpyruvic acid, $\mathrm{N}$-acetyl-vanilalanine, and $\mathrm{N}$-acetyl-tyrosine have also been discovered (Figure 1B). This finding is useful, as the detection of patients in normal screening programs for inborn errors of metabolism depends on finding an elevation of urinary VLA as the only abnormality detected, and thus the diagnosis may be easily missed.

Plasma AADC activity in symptomatic patients usually ranges from undetectable to $8 \%$ of normal. Carriers usually have intermediate AADC levels. ${ }^{1}$ Most carriers are clinically asymptomatic, although there has been a higher reporting rate of psychiatric manifestations. ${ }^{18}$
The CSF findings are not completely specific to AADC deficiency. Patients with a suspected defect in pyridoxal phosphate metabolism were found to have a similar CSF neurotransmitter metabolite profile. ${ }^{19}$

It is important to ensure that the patient tested is not receiving L-dopa therapy, as this can lead to a significant increase in 3-OMD. Dopamine $\beta$-hydroxylase deficiency can similarly cause an increase in 3-OMD; however, these cases do not present with neurological problems in childhood, and serotonin or dopamine levels are not deficient.

\section{Genetic analysis}

The $A A D C$ or dopa decarboxylase gene (DDC) lies on chromosome 7p12.1-p12.3 (Figure 3). The DDC gene comprises 15 exons spanning more than $85 \mathrm{~kb} .{ }^{20}$ Comparison of the amino acid sequence of DDC across species appears to indicate that the enzyme is an evolutionarily conserved molecule. The conserved amino acids are residues 267-317, which surround the pyridoxal phosphate binding site, ${ }^{21,22}$ as well as residues 64-155 and 182-204 that are important for the enzyme's catalytic function.

The majority of mutations reported are private mutations, although the splice site mutation IVS6+4A $>$ T may be a founder mutation in Southern Chinese individuals. In the study by Brun et al, ${ }^{9}$ the substitution mutation in Intron 6 , IVS6+4A $>\mathrm{T}$ was by far the most common mutation, followed by S250F, G102S, and R462P. ${ }^{9}$ The S250F mutation is particularly of interest, as this mutation may impact pyridoxal phosphate binding, and patients with this mutation may be particularly responsive to L-dopa and high-dose vitamin B6. ${ }^{22}$

AADC deficiency is an autosomal recessive condition and recurrence in families where both parents are carriers is one in four for every pregnancy. Prenatal diagnosis is possible if the mutations are identified. Preimplantation genetic diagnosis using amplification refractory mutation system-quantitative polymerase chain reaction on blastomere biopsies of day 3 cleavage-stage embryos has been performed in a Taiwanese family with unaffected live birth achieved. ${ }^{24}$ This was a family that had two consecutive abortions due to affected pregnancies ascertained by prenatal diagnosis after natural conception.

\section{Treatment options}

To date, since the majority of reported cases of AADC deficiency have been severe, the response to clinical treatment has been discouraging, as overall outcome has been relatively poor. In patients with a milder phenotype, however, there have been significant improvements in motor control and resolution of movement disorder such as dystonia. 


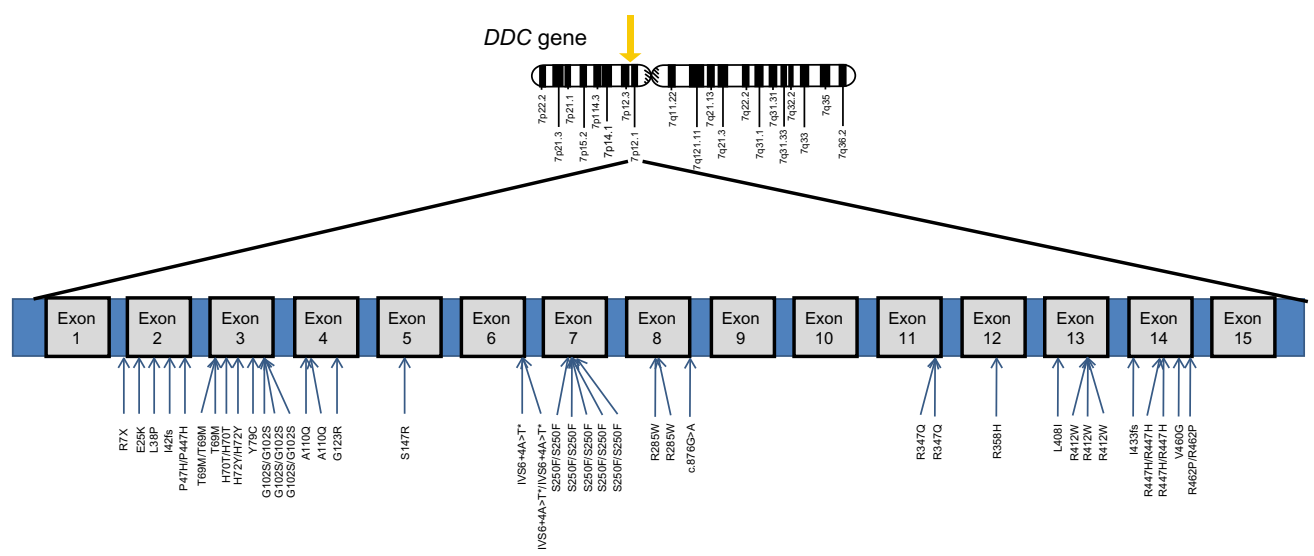

Figure 3 Distribution of known mutations in AADC or DDC showing mutation hotspot at splice site IVS6+4A $>$ T among Southern Chinese from Taiwan, Singapore, and Malaysia.

Notes: There was a slightly higher frequency of the S250F homozygous mutation in children of Italian and Arabic origin. Italicized mutations denote mutations in siblings. Arrows point to DDC gene location on chromosome 7p/2.I-7p/2.3. *Splice site IVS6+4A $>$ T.

Abbreviations: $A A D C$, aromatic amino-acid decarboxylase; DDC, dopa decarboxylase gene.

Early diagnosis and identification of the biochemical defect is therefore imperative to optimize the function of children that may see significant changes in abilities with appropriate treatment. The aim of treatment is to correct the neurotransmitter abnormalities and to potentiate enzyme activity with cofactor supplementation.

\section{Decreasing enzymatic degradation}

Depending on the severity of the enzyme deficiency, residual enzyme function can be optimized by targeting the monoamine oxidase that breaks down the AADC enzyme. Monoamine oxidase inhibitors (MAOI) such as selegiline and tranylcypromine are nonselective inhibitors that optimize the amount of functional AADC enzyme. Significant concerns exist about the interactions of MAOI with sympathomimetic drugs and tyramine-containing foods that could predispose an individual to hypertensive crises. However, we have noticed that our patients who are on MAOI have continued to consume foods such as soy sauce and other fermented foods that have higher levels of tyramine without detriment. It is nonetheless prudent to maintain vigilance with use of medications such as selective serotonin reuptake inhibitors (SSRIs) and tricyclic antidepressants.

Selegiline dose recommendations are usually based on adult dosing as MAOI are not usually recommended for children. Selegiline doses of $0.03-1.5 \mathrm{mg} / \mathrm{kg} /$ day were used according to the JAKE database. ${ }^{9}$ Tranylcypromine doses $0.2 \mathrm{mg} / \mathrm{kg} /$ dose every $8-12$ hours is recommended.

\section{Dopaminergic treatment}

Dopamine agonists are central to the treatment of AADC deficiency since the majority of motor symptoms are derived from central dopamine deficiency. These exist either as ergot and nonergot derived forms. Ergot derivatives such as pergolide and bromocriptine have been used effectively in the past, although there are some concerns about pericardial, pleuropulmonary, or retroperitoneal fibrosis when used in the long term. Nonergot dopamine agonists such as ropinirole and pramipexole are alternatives that may be considered, although the efficacy may not be as robust as for the ergot forms based on anecdotal reports of loss of motor abilities after changing from an ergot to a nonergot form of dopamine agonist. This may be due to the ergot forms of dopamine agonists having D1 receptor effects in addition to D2 agonist effects. Initiation of dopaminergic agonists should be performed cautiously and at low doses. Side effects such as dyskinesias and irritability should be watched out for. In addition, nonselective dopamine agonists such as bromocriptine may have some serotonergic effects as well. Bromocriptine is usually given at doses of $0.125-0.25 \mathrm{mg} / \mathrm{kg} /$ day in three divided doses and can be titrated up to $0.75 \mathrm{mg} / \mathrm{kg} /$ day in three divided doses. Pergolide should be given at a very low starting dosage of $0.006 \mathrm{mg} / \mathrm{kg} /$ day twice a day.

L-dopa, when used, needs to be started at very low doses and increased gradually in order to prevent complications of receptor hypersensitivity in severe cases of AADC deficiency. Most patients respond suboptimally to L-dopa, and strong responses have been reported in those who have mutations that affect the binding of L-dopa to AADC. ${ }^{9}$ Response to L-dopa may be dependent on the site of the mutations on the $A A D C$ gene. For example, the Ser250 site is not essential for catalytic activity of the AADC enzyme, but the S250F mutation causes a sevenfold decrease in catalytic activity through conformational changes in the protein. 
Table I Diagnostic parameters for AADC deficiency

\begin{tabular}{|c|c|}
\hline Clinical features & $\begin{array}{l}\text { Percentage of patients in the } \\
\text { JAKE database of } 78 \text { patients }\end{array}$ \\
\hline \multicolumn{2}{|l|}{ Motor manifestations: } \\
\hline Hypotonia & 95 \\
\hline Oculogyric crises & 86 \\
\hline Developmental retardation & 63 \\
\hline Dystonia & 53 \\
\hline Hypertonia & 44 \\
\hline Feeding and swallowing & 42 \\
\hline \multicolumn{2}{|l|}{ difficulties } \\
\hline Dysarthria & 41 \\
\hline Ptosis & 39 \\
\hline Hypokinesia & 32 \\
\hline Poor head control & 28 \\
\hline Athethosis & 27 \\
\hline Poor eye fixation & 26 \\
\hline Chorea & 22 \\
\hline \multicolumn{2}{|l|}{ Autonomic signs: } \\
\hline Sweating & 65 \\
\hline Hypersalivation & 41 \\
\hline Temperature instability & 29 \\
\hline Blood pressure and/or & 15 \\
\hline \multicolumn{2}{|l|}{ heart rate disturbances } \\
\hline Hypoglycemia & $<1 \%(2$ cases $)$ \\
\hline \multicolumn{2}{|l|}{ Other signs: } \\
\hline Insomnia & 37 \\
\hline Irritability & 35 \\
\hline Nasal congestion & 31 \\
\hline
\end{tabular}

Biochemical parameters

Cerebrospinal fluid:

5-hydroxyindoleacetic acid level $\downarrow$

Homovanillic acid level $\downarrow$

3-methoxy-4-hydroxyphenylglycol level $\downarrow$

L-dopa level $\uparrow$

3-O-methyldopa level $\uparrow$

5-hydroxytryptophan level $\uparrow$

Pterin levels normal

Plasma:

3-O-methyldopa level $\uparrow$

Enzyme assay (see below)

Urine:

Vanillactic acid level may be $\uparrow$

Vanilpyruvic acid may be $\uparrow$

$\mathrm{N}$-acetyl-vanilalanine may be $\uparrow$

$\mathrm{N}$-acetyl-tyrosine may be $\uparrow$

Paradoxical hyperdopaminuria

Enzyme analysis

Plasma AADC activity usual range: undetectable to $8 \%$ of normal

Genetic analysis of DDC (available for $\mathbf{4 9}$ out of the $\mathbf{7 8}$ patients in the JAKE database)

IVS6+4A $>\mathrm{T}$ (most common)

$\mathrm{S} 250 \mathrm{~F}$

GI02S

R462P

R347Q/RI60 W

R347Q/R358H

R285W/IVS6+4A $>T$
Table I (Continued)

$\mathrm{R} 4 \mathrm{I} 2 \mathrm{~W} / \mathrm{IVS} 6+4 \mathrm{~A}>\mathrm{T}$

$\mathrm{R} 447 \mathrm{H}$

L408I/R7

GI23R

$\mathrm{R} 358 \mathrm{H}$

T69M

AIIOQ

SI47R

$\mathrm{P} 47 \mathrm{H}$

L38P

c. $876 \mathrm{G}>\mathrm{A}$

E25K/V460G

Abbreviations: AADC, aromatic amino-acid decarboxylase; DDC, dopa decarboxylase gene.

In some cases, L-dopa without decarboxylase inhibitors was started with the aim of stimulating the residual enzyme activity by providing an excess of substrate. The results seemed more encouraging, particularly when the L-dopa was combined with vitamin B6. In general, a trial of L-dopa should be considered in patients, but with caution as L-dopa may decrease in methylation capacity. A low dose L-dopa/carbidopa (100 mg/25 mg) tablet can be administered once a day, titrating up to three times a day. Objective testing of CSF neurotransmitters should be considered after the L-dopa/carbidopa dose has been established for approximately 2 weeks, with timing of the CSF sampling after taking the L-dopa/carbidopa dose.

\section{Serotonergic treatment}

SSRIs and serotonin agonists may be considered when addressing serotonin deficiency. SSRIs must be used with caution in patients who are on MAOI. Monitoring responses to serotonergic treatment is more challenging than for dopaminergic treatment, as nonmotor manifestations are harder to quantify.

\section{Cofactor and other supplementation}

Pyridoxine (vitamin B6) is the precursor of pyridoxal phosphate, which is a cofactor of AADC. Reduced levels of pyridoxine may not only be required for AADC enzymatic activity, but may also lead to a loss of AADC enzymes in the cells. ${ }^{25}$ Supplementation with either pyridoxine or pyridoxal phosphate is recommended to augment residual AADC activity. It is not certain how beneficial high doses of pyridoxine are in patients with AADC deficiency, but the pyridoxine dose should not exceed $200 \mathrm{mg} / \mathrm{kg} /$ day. Specific evaluation of the efficacy of pyridoxine supplementation can be performed by taking neurotransmitter, folate metabolites, and s-adenosyl methionine levels before and after supplementation. 
Additionally, certain mutations may be associated with better responses with pyridoxine administration, such as the $\mathrm{S} 250 \mathrm{~F}$ mutation, for which pyridoxine may increase the expression level of the AADC enzyme. ${ }^{23}$

Secondary central folate (tetrahydrofolate) deficiency may be seen in AADC deficiency due to methylation of accumulated L-dopa. Empirical treatment in other secondary folate deficiencies has been associated with improved control of movement disorders. A daily folinic acid dose of $10-20 \mathrm{mg}$ has been recommended. Adequate dietary intake of folate is also recommended. ${ }^{24}$

\section{Symptomatic treatment}

Anticholinergic therapy can ameliorate dystonic crises and may also reduce excessive sweating through sympathomimetic effects on sweat glands. Trihexyphenidyl and amantadine are anticholinergics that have been used with effect in some patients. It is also thought that anticholinergic therapy may also smooth out some of the motor fluctuations experienced by patients.

Seizures are rare in AADC deficiency and standard antiepileptic therapy may be considered.

Hypoglycemia has been reported in severe cases as a result of a relative lack of catecholamines that act as antiinsulinergic hormones. Newly suspected AADC deficiency cases that have a more severe phenotype should have fasting glucose levels checked, especially if the child has had fluctuations in symptoms associated with fasting. Prevention of hypoglycemia with dietary management (for example, corn starch) may be critical in infancy. Monitoring of blood glucose levels may be necessary, particularly if the child has a gastrointestinal illness. ${ }^{27}$

OGCs are usually a result of the low central dopamine state and the frequency of these OGCs may decrease with the optimization of dopaminergic treatments.

\section{Clinical trials}

Gene therapy is the holy grail of AADC therapy; however, gene therapy may be challenging because of the need to deliver the gene and vector to the central nervous system. There is a recent report of $A A D C$ gene therapy with intracerebral infusion of AAV2-hAADC viral vector with evidence of motor improvements and increased CSF dopamine and serotonin levels following gene transfer. ${ }^{28}$

\section{Treatment response}

With regard to the first pair of patients diagnosed with AADC deficiency (ie, Hyland et al), ${ }^{2,11}$ treatment with either bromocriptine or tranylcypromine ceased the abnormal eye movements, and tranylcypromine also improved the muscle tone. The plasma norepinephrine and whole blood serotonin levels were improved. Combined treatment with pyridoxine, bromocriptine, and tranylcypromine led to sustained improvement in tone and voluntary movements.

A study by Abeling et $\mathrm{al}^{29}$ suggested that the degree of enzyme deficiency may not be uniform in different organ systems, and that AADC may be more active in the renal proximal tubular epithelial cells of the patients. In the kidney, the renal dopamine system is essential in sodium transport, thus contributing to the control of water balance and blood pressure. Most of the patients diagnosed earlier did not have hypertension, and this seems to suggest that AADC may be normally functioning in the kidneys and adrenals, or that there is an underlying alternative biochemical route in these tissues.

Some clinical heterogeneity has been observed among patients with AADC deficiency with regard to their response to therapy. There has been an association of more severe symptoms and poorer response to therapy in females compared with males. This has been attributed to a higher dependence on the dopamine system in females. Serendipitously, our two patients with AADC deficiency were both females with mild phenotype who improved remarkably with treatment; both were wheelchair bound and with treatment had improved independent mobility, disappearance of dystonia and OGCs was evident, but there was residual mild autonomic dysfunction such as drooling and sleep disturbance.

It is hard to draw genotype-phenotype correlation of treatment response based on mutation types. The most common IVS6+4A $>\mathrm{T}$ splicing mutation found in Chinese individuals was seen in approximately one-third of patients in the JAKE database. Of these 25 patients, 15 had documented treatment response, and seven patients showed improvement of a variety of symptoms, including a decrease in OGCs, decreased dystonia, speech difficulties, insomnia, and temperature instability. These had been treated with a combination of MAOI (selegiline), dopamine agonists (either bromocriptine or pergolide), pyridoxine, and folinic acid. Four of these responders had been given L-dopa as well. Others $(8 / 15 ; 53.3 \%)$ with either homozygous or heterozygous IVS6+4A $>$ T mutation did poorly and did not respond to the cocktail of dopamine agonists, anticholinergics, MAOI, and pyridoxine.

A recent report identified a child with $\mathrm{R} 347 \mathrm{Q} / \mathrm{R} 160 \mathrm{~W}$ mutations who had AADC enzyme kinetics tested, revealing a sixfold increase in the affinity for L-dopa suggesting 
a modification in the substrate binding site. ${ }^{30}$ This patient was treated with L-dopa and pyridoxine and showed marked improvement in terms of quality of life, fatigability, and paroxysmal eye movements (OGCs). This illustrates the fact that certain mutations may significantly affect enzyme kinetics, and substrate supplementation (L-dopa) may result in markedly improved clinical function.

In a few patients, the response to therapy was initially positive, followed by a plateau, then subsequent deterioration. A set of three siblings with the G102S homozygous mutation appeared to have a good response of hypersalivation, sweating, tremor, dysarthria, and OGCs with use of L-dopa, but they then appeared to plateau in response and subsequently deteriorated; ${ }^{31}$ this may suggest tolerance or saturation at the receptors. On the other hand, AADC deficiency, like many other metabolic disorders, may be progressive and patients may be deteriorating despite optimal therapy.

The outcomes of eleven patients reported by Swoboda et $\mathrm{al}^{32}$ illustrate the suboptimal clinical outcome of patients with AADC deficiency. All remained nonverbal and demonstrated severe axial hypotonia and complete functional dependence on others for activities of daily living. These patients also demonstrated multisystemic involvement due to autonomic dysfunction, motor difficulties, and problems with sleep, attention, emotional regulation, and cognition.

The use of neurotransmitter receptor agonists and drugs that block enzyme breakdown of neurotransmitters seems to still be inadequate in view of the marked complexity of the receptor systems of both dopamine and serotonin in the central nervous system. The replacement of AADC via direct enzyme replacement, stem cell strategies, or gene therapy to allow a fully functional and optimally regulated gene into widely dispersed target cells still seems to be a difficult challenge.

Another possibility of the direct replacement of dopamine and serotonin into the ventricular system via a pump device that can allow almost continuous delivery appears promising, but remains technically challenging.

At present, supportive medical care and the judicious use of therapeutic agents appear to form the best option for patients.

\section{Our experience with AADC deficiency}

A pair of sisters had been diagnosed with AADC deficiency in our institution when they were 13 years and 10 years of age. ${ }^{33}$ Both siblings are now 21 years old and 18 years old, having been followed up for the past 8 years. Antenatal history was unremarkable for both sisters. Their parents were nonconsanguineous and there was no family history of neurological disorders or developmental delay.

The older sister was observed to have hypotonia and gross motor delay since 3 months of age. She was born at 35 weeks' gestation via emergency Cesarean section for fetal distress and cord prolapse. Her birth weight was $2.25 \mathrm{~kg}$.

When she presented with poor head control, she was also noticed to have bilateral ptosis and significant drooling. She did not have any episodes of hypoglycemia, seizures, or obvious OGCs. She developed head control at about 12 months, sat independently at 18 months, and walked at 2 years and 9 months of age. She never crawled. Cognitive delays were also noted later and intelligence quotient assessment showed a borderline intelligence quotient of $83 \pm 7$ at 6 years of age. There were no swallowing problems, although she drooled frequently.

She was in an early intervention program, but subsequently attended mainstream primary school, where she struggled with academic performance. During her evaluation, she was described to have fluctuating motor power, worse when she was tired, and she required multiple 10- to 15-minute naps throughout the day to regain her motor abilities. Her motor abilities could be sustained for about 20 minutes to 30 minutes at best. She was able to walk independently 10 meters to 20 meters at best, and on some days was only able to walk for 2 meters to 3 meters.

Clinical examination at presentation showed a cheerful and well grown child, with both weight and height at the 25 th percentile. She had small hands and feet. She was able to walk independently, tending to list to one side because of mild truncal dystonia. There was bilateral ptosis, with difficulty sustaining upward gaze for more than 15 seconds. She did not have ophthalmoplegia. She had hypernasal speech, but normal movement of the palate. Intermittent drooling was present. There was no facial asymmetry; pupils were reactive and fundoscopy was normal. Hearing was normal.

Tone was slightly decreased. Power was at least grade 4 in all four limbs, but there was difficulty in maintaining the effort. The degree of weakness was greatest in the neck extensors. Deep tendon reflexes were increased $(3+)$ in the lower limbs and $2+$ in the upper limbs. The plantar reflexes were upgoing bilaterally. No contractures were present. Her gait was wide based with marked proximal weakness (flexed hips and knees and flexion of trunk). Speech was mildly dysarthric but there were no cerebellar signs. Sensation and proprioception was intact. 
The diagnosis of AADC deficiency was made when she had abnormal CSF neurotransmitter levels in July 2005. Incidentally, the procedure of sedation and spinal tap triggered off her first OGC. Her CSF neurotransmitters were typical of AADC deficiency - the level of 5HIAA was decreased at $13 \mathrm{nmol} / \mathrm{L}$ (normal reference range $67-189 \mathrm{nmol} / \mathrm{L}$ ); HVA was decreased at $64 \mathrm{nmol} / \mathrm{L}(167-563 \mathrm{nmol} / \mathrm{L})$; and, 3-OMD was increased at $310 \mathrm{nmol} / \mathrm{L}(<100)$. The concentrations of $\mathrm{BH}_{4}$ and neopterin in CSF were within the reference ranges. Her plasma AADC levels were assayed and the diagnosis of AADC deficiency was confirmed. She was started on selegiline and pyridoxine. The choice of a MAOI as a first treatment was because of her milder phenotype and likely higher amount of residual AADC enzyme that might respond well to MAOI therapy. There was clear immediate improvement of motor strength, more sustained motor power, and she was able to walk for longer distances soon after starting medications. Bromocriptine was also started subsequently to optimize her motor function. She was subsequently able to walk independently for several hours at a time and rarely needed to nap during the day to regain motor strength. She is currently on bromocriptine $5 \mathrm{mg}$ twice a day, selegiline $5 \mathrm{mg}$ three times a day, pyridoxine $100 \mathrm{mg}$ twice a day, and folinic acid $3.75 \mathrm{mg}$ once a day.

On these doses of medication, which have been stable for the past 8 years, her gait was still broad based but had no restriction in motor activities and she would participate in regular physical education classes in school. She has continued well academically and is currently in an institute of technical education for training in office administration. She continued to have mildly dysarthric and hypernasal speech, as well as drooling, which had resulted in bullying during her teenage school years. Despite these difficult social adjustments, she maintained high spirits and mood and did not have evidence of depression or dysphoria. There were occasional instances where she has episodes of "exhaustion" and difficulty in initiating movements, as well as increased sleep requirements and naps during the day. As these episodes were short, there was no change in medication dosage. She had refused any further spinal taps for assessment of neurotransmitter levels because of her previous experience with an OGC triggered by the procedure.

Her younger sister had a similar but slightly more severe clinical presentation. She was born full term via elective Cesarean section with good Apgar scores. Her birth weight was $2.77 \mathrm{~kg}$. She was also observed to have motor delay from 3 months of age. Cognitive delays were also present. During the first year of life, she was noticed to have postural deformity of the left leg. She was not able to ambulate independently prior to specific treatment of the AADC deficiency. There was no swallowing problem, although she drooled frequently. Similar to her older affected sister, she had marked fluctuation in motor power, with definite need to sleep short naps throughout the day to regain her motor abilities. She also had OGCs that would occur during periods of fatigue. These were not recognized as OGCs, as there was only eye deviation.

Her developmental milestones were delayed. She was able to sit independently after 2 years of age. She had never crawled. Prior to treatment of AADC deficiency, she was unable to walk without assistance. Gait was markedly unstable because of limb dystonia (worse on the left than the right lower limb). She was mainly wheelchair bound. At the age of 10 years when she first presented to our institution, she was able to write and draw, and feed herself. She was able to speak full sentences, but her articulation was not clear. Cognitively, she was able to do simple arithmetic and to spell words. She previously attended special education.

She had splitting of the tibialis posterior and tendon transfer in the left foot when she was 9 years of age, to address the problem of contracture of the Achilles tendon.

Clinical examination at presentation at 10 years old showed a cheerful and well grown child, with weight and height at 25th percentile. She was able to walk only with maximal assistance, with a broad-based gait and marked limb and truncal dystonia. She was otherwise wheelchair bound, with poor neck and trunk control, and would sit with a curved back in her chair. There was mild bilateral ptosis. She was able to sustain an upward gaze for only 10 seconds. There were episodes of eye deviation that were confirmed later to be mild OGCs. There was dystonia of the left ankle and an equinovarus deformity of her left ankle. Tone was increased in both ankles. Deep tendon reflexes were more brisk in the lower limbs than in the upper limbs. Plantar reflexes were upgoing bilaterally. Speech was dysarthric, but there were no other cerebellar signs.

Her plasma AADC levels were assayed when she was 10 years old and the diagnosis of AADC deficiency was confirmed. Other investigations included magnetic resonance imaging of the brain, which was normal. She was started on selegiline and pyridoxine. There was improvement in hypersalivation as well as truncal control. There was no clear improvement in effort tolerance or the frequency of OGC. Bromocriptine was also started, and with intensive daily physiotherapy, she improved markedly and progressed from wheelchair dependency to independent ambulation. 
She subsequently enrolled in mainstream primary school at the age of 11 years. She was able to cope with mainstream education and recently passed the national Primary School Leaving Examination and was able to continue in mainstream secondary school education. She is currently on bromocriptine $5 \mathrm{mg}$ three times a day, selegiline $5 \mathrm{mg}$ twice a day, pyridoxine $100 \mathrm{mg}$ twice a day, and folinic acid $3.75 \mathrm{mg}$ once a day. Like her sister, she has not required any dose changes over the past 8 years as she had stable motor abilities, minimal requirement for naps during school days, and did not have any recurrence of dystonia. Her mood was usually extremely cheerful and there was no evidence of dysphoria or depression. Clinically, she had a broad-based gait, but was able to walk and run stably. She had low axial tone, but had good power in all limbs. Deep tendon reflexes in the lower limbs were brisk. She had mild dysarthria and hypernasal speech, as well as occasional drooling.

To our knowledge, these siblings represent the mildest known phenotype of AADC deficiency. Unlike the other patients with IVS6+4A $>$ T mutations, they had milder clinical symptoms that responded well to medical therapy, without the need for dose titration over a period of observation of 8 years. These two girls also challenge the presumption of a more severe phenotype in females with AADC deficiency.

\section{Conclusion}

AADC deficiency is a rare pediatric neurotransmitter disorder that presents with hypotonia, encephalopathy, movement disorder, OGCs, and fluctuating motor symptoms from infancy. While most known cases have a relatively severe presentation with marginal response to medical therapy, we have observed patients with mild symptoms correctable with dopamine agonists and MAOI. These cases illustrate the need for a good evaluation for patients with suspected neurotransmitter disorders, as there is a potential for life-changing therapy in individuals with a milder phenotype.

\section{Disclosure}

The authors report no conflicts of interest in this work.

\section{References}

1. Verbeek MM, Geurtz PB, Willemsen MA, Wevers RA. Aromatic L-amino acid decarboxylase enzyme activity in deficient patients and heterozygotes. Mol Genet Metab. 2007;90(4):363-369.

2. Hyland K, Surtees RA, Rodeck C, Clayton PT. Aromatic L-amino acid decarboxylase deficiency: clinical features, diagnosis, and treatment of a new inborn error of neurotransmitter amine synthesis. Neurology. 1992;42(10):1980-1988.

3. Carlsson A, Lindqvist M, Magnusson T, Waldeck B. On the presence of 3-hydroxytyramine in brain. Science. 1958;127(3296):471.
4. Dahlstroem A, Fuxe K. Evidence for the existence of monoaminecontaining neurons in the central nervous system. I. Demonstration of monoamines in the cell bodies of brain stem neurons. Acta Physiol Scand Suppl. 1964;Suppl 232:1-55.

5. Groenewegen HJ. The basal ganglia and motor control. Neural Plast. 2003;10(1-2):107-120.

6. Berger M, Gray JA, Roth BL. The expanded biology of serotonin. Annu Rev Med. 2009;60:355-366.

7. Sternbach H. The serotonin syndrome. Am J Psychiatry. 1991;148(6): 705-713.

8. Fiumara A, Bräutigam C, Hyland K, et al. Aromatic L-amino acid decarboxylase deficiency with hyperdopaminuria. Clinical and laboratory findings in response to different therapies. Neuropediatrics. 2002;33(4):203-208.

9. Brun L, Ngu LH, Keng WT, et al. Clinical and biochemical features of aromatic L-amino acid decarboxylase deficiency. Neurology. 2010; 75(1):64-71.

10. JAKE database. Available at: http://www.biopku.org/bioPKU_databasesJAKE.asp. Accessed July 11, 2013.

11. Hyland K, Clayton PT. Aromatic amino acid decarboxylase deficiency in twins. J Inherit Metab Dis. 1990;13(3):301-304.

12. Kurian MA, Gissen P, Smith M, Heales S Jr, Clayton PT. The monoamine neurotransmitter disorders: an expanding range of neurological syndromes. Lancet Neurol. 2011;10(8):721-733.

13. Assmann B, Surtees R, Hoffmann GF. Approach to the diagnosis of neurotransmitter diseases exemplified by the differential diagnosis of childhood-onset dystonia. Ann Neurol. 2003;54 Suppl 6:S18-S24.

14. Lee WT, Weng WC, Peng SF, Tzen KY. Neuroimaging findings in children with paediatric neurotransmitter diseases. J Inherit Metab Dis. 2009;32(3):361-370.

15. Abdenur JE, Abeling N, Specola N, et al. Aromatic l-aminoacid decarboxylase deficiency: unusual neonatal presentation and additional findings in organic acid analysis. Mol Genet Metab. 2006;87(1):48-53.

16. Wassenberg T, Willemsen MA, Geurtz PB, et al. Urinary dopamine in aromatic L-amino acid decarboxylase deficiency: the unsolved paradox. Mol Genet Metab. 2010;101(4):349-356.

17. Wassenberg T, Monnens LA, Geurtz BP, Wevers RA, Verbeek MM, Willemsen MA. The paradox of hyperdopaminuria in aromatic L-amino acid deficiency explained. JIMD Rep. 2012;4:39-45.

18. Paediatric Movement Disorders. Fernández-Alvarez E, Arzimanoglou A, Tolosa E, editors. France: John Libbey Eurotext Ltd, 1st edition; 2005.

19. Footitt EJ, Heales SJ, Mills PB, Allen GF, Oppenheim M, Clayton PT. Pyridoxal 5'-phosphate in cerebrospinal fluid; factors affecting concentration. J Inherit Metab Dis. 2011;34(2):529-538.

20. Sumi-Ichinose C, Ichinose H, Takahashi E, Hori T, Nagatsu T. Molecular cloning of genomic DNA and chromosomal assignment of the gene for human aromatic L-amino acid decarboxylase, the enzyme for catecholamine and serotonin biosynthesis. Biochemistry. 1992;31(8):2229-2238.

21. Ichinose H, Kurosawa Y, Titani K, Fujita K, Nagatsu T. Isolation and characterization of a cDNA clone encoding human aromatic L-amino acid decarboxylase. Biochem Biophys Res Commun. 1989;164(3): 1024-1030.

22. Maras B, Dominici P, Barra D, Bossa F, Voltattorni CB. Pig kidney 3,4-dihydroxyphenylalanine (dopa) decarboxylase. Primary structure and relationships to other amino acid decarboxylases. Eur J Biochem. 1991;201(2):385-391.

23. Montioli R, Oppici E, Cellini B, Roncador A, Dindo M, Voltattorni CB. S250F variant associated with aromatic amino acid decarboxylase deficiency: molecular defects and intracellular rescue by pyridoxine. Hum Mol Genet. 2013;22(8):1615-1624.

24. Hyland K. Inherited Disorders Affecting Dopamine and Serotonin: Critical Neurotransmitters Derived from Aromatic Amino Acids. J Nutr. 2007;137(6):15685-15725.

25. Kuo SJ, Ma GC, Chang SP, et al. Preimplantation and prenatal genetic diagnosis of aromatic L-amino acid decarboxylase deficiency with an amplification refractory mutation system-quantitative polymerase chain reaction. Taiwan J Obstet Gynecol. 2011;50(4):468-473. 
26. Allen GF, Neergheen V, Oppenheim M, et al. Pyridoxal $5^{\prime}$-phosphate deficiency causes a loss of aromatic L-amino acid decarboxylase in patients and human neuroblastoma cells, implications for aromatic L-amino acid decarboxylase and vitamin $\mathrm{B}(6)$ deficiency states. J Neurochem. 2010;114(1):87-96.

27. Pons R, Ford B, Chiriboga CA, et al. Aromatic L-amino acid decarboxylase deficiency: clinical features, treatment, and prognosis. Neurology. 2004;62(7):1058-1065.

28. Hwu WL, Muramatsu S, Tseng SH, et al. Gene therapy for aromatic L-amino acid decarboxylase deficiency. Sci Transl Med. 2012;4(134) $134 \mathrm{ra} 61$.

29. Abeling NG, Bräutigam C, Hoffmann GF, et al. Pathobiochemical implications of hyperdopaminuria in patients with aromatic L-amino acid decarboxylase deficiency. J Inherit Metab Dis. 2000;23(4):325-328.
30. Barth M, Serre V, Hubert L, et al. Kinetic analyses guide the therapeutic decision in a novel form of moderate aromatic acid decarboxylase deficiency. JIMD Rep. 2012;3:25-32.

31. Chang YT, Sharma R, Marsh JL, et al. Levodopa-responsive aromatic L-amino acid decarboxylase deficiency. Ann Neurol. 2004;55(3):435-438.

32. Swoboda KJ, Saul JP, McKenna CE, Speller NB, Hyland K. Aromatic L-amino acid decarboxylase deficiency: overview of clinical features and outcomes. Ann Neurol. 2003;54 Suppl 6:S49-S55.

33. Tay SK, Poh KS, Hyland K, et al. Unusually mild phenotype of AADC deficiency in 2 siblings. Mol Genet Metab. 2007;91(4):374-378.

Pediatric Health, Medicine and Therapeutics

\section{Publish your work in this journal}

Pediatric Health, Medicine and Therapeutics is an international, peerreviewed, open access journal publishing original research, reports, editorials, reviews and commentaries. All aspects of health maintenance preventative measures and disease treatment interventions are addressed within the journal. Practitioners from all disciplines are invited to submit their work as well as healthcare researchers and patient support groups. The manuscript management system is completely online and includes a very quick and fair peer-review system. Visit http://www.dovepress.com/ testimonials.php to read real quotes from published authors.

Submit your manuscript here: http://www.dovepress.com/pediatric-health-medicine-and-therapeutics-journal 